\title{
Effect of Disorder on the Critical Temperature of a Dilute Hard-Sphere Gas
}

\author{
M. C. Gordillo ${ }^{1}$ and D. M. Ceperley ${ }^{2}$ \\ ${ }^{1}$ Departament de Física i Enginyeria Nuclear, Campus Nord B4-B5, Universitat Politècnica de Catalunya, \\ E-08034 Barcelona, Spain \\ ${ }^{2}$ Department of Physics and NCSA, University of Illinois, Urbana-Champaign, Illinois 61801
}

(Received 27 July 2000)

\begin{abstract}
We have performed path integral Monte Carlo calculations to determine the effect of quenched disorder on the superfluid density of a dilute 3D hard-sphere gas. The disorder was introduced by locating hard cylinders randomly inside the simulation cell. Our results indicate that the disorder does not strongly affect the superfluid critical temperature. There is a reduction of $\rho_{s} / \rho$ with increasing disorder and with excluded volume for similar disorders and a possible change of universality class (as evidenced by the correlation length exponent) at high disorder. Comparison to experiments of helium in Vycor is made.
\end{abstract}

PACS numbers: $67.40 . \mathrm{Hf}$

Recently there have been several theoretical studies of the superfluid transition (Bose condensation) of a system of homogeneous hard spheres. For certain properties the model of hard spheres can describe the Bose-Einstein condensates of alkali metal atoms and liquid ${ }^{4} \mathrm{He}$. The ratio of the superfluid transition temperature to that of an ideal gas $T_{c} / T_{c 0}$ was determined with numerical calculations [1] to be larger than unity over a wide range of densities. The enhancement has been confirmed by several other studies [2,3], however the magnitude of the enhancement as a function of density is still controversial. It has also been proposed that $T_{c}$ can be enhanced with respect to an ideal gas by the introduction of quenched (i.e., static) disorder [4]. Experimental results for ${ }^{4} \mathrm{He}$ in Vycor show that, for some concentrations, the enhancement is as large as $300 \%$. Here, we study numerically how a low density hard-sphere gas is affected by random geometry. Although there have been many calculations for disordered lattice models $[5,6]$, to our knowledge, there are no other calculations tackling this problem in the continuum.

Both helium gas and hard-sphere systems fall into the universality class in which the critical exponents of the superfluid fraction $\varphi$ and the correlation length $\nu$ satisfy $\varphi=-\nu=-0.67$. In this class, the critical exponent of the specific heat, $\alpha$ is negative and according to the Harris criterion [7], the presence of weak uncorrelated disorder will not change the exponents. To examine both this change in $\nu$ and the possible variation in $T_{c}$ with the presence of quenched disorder, one can observe the behavior of helium when absorbed in a network of porous silica [8-13]. This material is produced with various porosities. For example, in aerogel the volume of the voids in the system is around $90 \%-95 \%$, in xerogel or porous gold it is $60 \%$, and in Vycor the porosity is $\sim 30 \%$. Experimentally, it is found that the introduction of disorder decreases both the critical temperature and the fraction of the helium atoms that decouple from the oscillator measurement of the superfluid. In addition, the critical exponents can be changed: for samples with large porosity, $\nu$ has a value greater than of the bulk helium case $[10,11]$. However, in
Vycor [10] and porous gold [14], the exponents are the same as in bulk ${ }^{4} \mathrm{He}$.

Since a system of homogeneous hard spheres belongs to the same universality class as bulk liquid ${ }^{4} \mathrm{He}$, we tried to understand the changes in the critical temperature and critical exponents in silica gels by performing path integral Monte Carlo (PIMC) calculations [15] for a system of hard spheres with quenched disorder. A simple model of disorder was constructed by placing, at random, $N_{c}$ hard cylinders parallel to the edges of the cubic periodic simulation cell. We used hard cylinders rather than a more realistic model of Vycor or aerogel, because such a model is much easier to treat computationally, in part because the absence of an attractive interaction means that there will not be a "dead layer" of ${ }^{4} \mathrm{He}$ next to the substrate which would impose a computational burden without participating in superflow. We sampled the positions of the cylinders by doing a simulation of a 2D hard quantum hard disk. One snapshot was used to place the cylinders along the $x$ axis, another independent snapshot for the $y$ axis, and a third for the $z$ axis. Thus there were always equal numbers of cylinders along each axis. This procedure generates a random placement of the cylinders which are more uniformly spread across the simulation cell than would be the case if each cylinder was inserted independently.

The hard spheres are chosen to have diameter $\sigma$ appropriate to ${ }^{4} \mathrm{He}(2.2 \AA)$ so as to make comparison straightforward, though we will also use dimensionless units. We demand that the hard spheres remain a distance $d$ away from the center of each of the cylinders. The diameters of the cylinders were varied to study the effect of their number and/or of the total excluded volume. We considered two densities: $\rho \sigma^{3}=0.0107$ and $1.07 \times 10^{-5}$. Here, $\rho=N_{s} / L^{3}, N_{s}$ is the number of hard spheres, and $L$ the length of the simulation cell. A summary of the conditions used is given in Table I. Each simulation was repeated for five different realizations of the disorder.

There are four dimensionless parameters characterizing this system in the thermodynamic limit: the density of hard spheres $\rho \sigma^{3}$, the fraction of excluded volume, an upper 
TABLE I. Different cylinder arrangements and simulation conditions used in this work. Systems II and IV have the same randomness (placement of the cylinders).

\begin{tabular}{|c|c|c|c|c|c|c|}
\hline System & $\rho \sigma^{3}$ & $\mu$ & $d / \sigma$ & $\rho_{s}(T=0) / \rho$ & $N_{c}$ & $N_{s}$ \\
\hline I & $1.07 \times 10^{-2}$ & 0.128 & 1 & $0.68 \pm 0.10$ & $\begin{array}{r}6 \\
12 \\
18 \\
24\end{array}$ & $\begin{array}{r}19 \\
54 \\
9 \\
153\end{array}$ \\
\hline II & $1.07 \times 10^{-2}$ & 0.128 & 1.5 & $0.74 \pm 0.05$ & $\begin{array}{r}3 \\
6 \\
9 \\
12\end{array}$ & $\begin{array}{r}23 \\
64 \\
118 \\
182\end{array}$ \\
\hline III & $1.07 \times 10^{-2}$ & 0.33 & 1.5 & $0.51 \pm 0.19$ & $\begin{array}{r}9 \\
15 \\
21 \\
30\end{array}$ & $\begin{array}{r}29 \\
62 \\
102 \\
174\end{array}$ \\
\hline IV & $1.07 \times 10^{-5}$ & 0.128 & 15 & $0.56 \pm 0.09$ & $\begin{array}{r}3 \\
6 \\
9 \\
12\end{array}$ & $\begin{array}{r}23 \\
64 \\
118 \\
182\end{array}$ \\
\hline
\end{tabular}

bound is $\mu=\pi N_{c}(d / L)^{2}$ (an upper bound because if two cylinders are close to each other, some of the excluded volume is counted twice); the ratio $d / \sigma$; and the ratio of the temperature to that of free boson transition temperature at the same density $\left(T_{c 0}=0.401 \mathrm{~K}\right.$ at $\rho \sigma^{3}=0.0107$, not taking into account excluded volume.)

First, let us examine how the precise geometry of the disorder affects the superfluid fraction. Figure 1 displays $\rho_{s} / \rho$, for system II, as a function of $T / T_{c 0}$. The superfluid density [15] is computed by calculating the mean squared

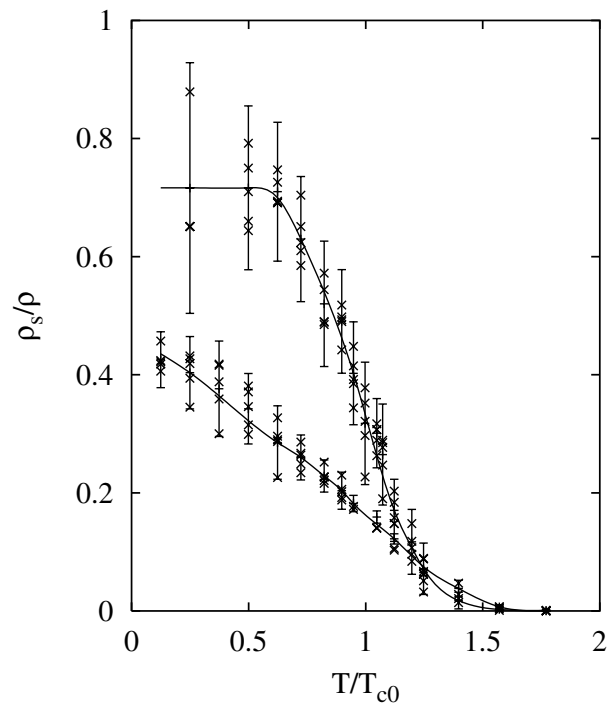

FIG. 1. The upper curve shows the superfluid fraction for system II with $N_{c}=9$ and $N_{s}=118$. The symbols correspond to individual realizations, and the curve with error bars to the disorder average. The error bars indicate one standard deviation. The lower curve shows results for an ideal gas. winding of the paths around the periodic boundary conditions. The crosses indicate the results for each disorder at a given temperature, and the lines are the smoothed averages of the superfluid fractions for the five cases. All the values of $\rho_{s} / \rho$ are within a standard deviation of the average values, indicating that the superfluid fraction does not depend much on the particular geometry chosen and that one can speak of a single $T_{c}$. Even though our systems are small they seem to be "self-averaging." The behavior of system II is typical of the other three geometries.

The data in the lower part of the figure were obtained by "switching off" the interactions between the hard spheres and keeping constant the rest of the simulation conditions, to gauge the importance of the interaction on the results. We refer to this as the "disordered" ideal gas. We did this for systems I, II, and III. It can be observed that when the hard-core interaction is turned off, the fraction of molecules belonging to the superfluid at low temperatures decreases by a factor of 2 . In the thermodynamic limit, the effect is even greater; the disorder suppresses the superfluidity of the ideal gas. This can be easily understood since, without interaction, there exists a lowest energy state, localized in the cavity with the largest volume, that will be occupied by all the bosons. Thus, when $N_{s} \rightarrow \infty$, one will have BEC but not superfluidity consistent with our computed superfluid densities.

Another effect we observe in our simulation is the reduction of superfluid density with increasing disorder defined in our system as the inverse mean free path of a classical hard sphere. That reduction can be seen in Fig. 2 for the different systems with $N_{s} \sim 60$. The tortuosity, which we define as the fraction of atoms participating in superflow at zero temperature, $\chi=\rho_{s}(T=0) / \rho$ given in Table I, is found to be independent of $N_{s}$ and $0.51 \leq \chi \leq 0.74$. These values are reasonably consistent with those measured in Vycor $\chi=0.76 \pm 0.01$ [8]. The greater the excluded volume (system III vs system II) for the same type of cylinders, the lower $\chi$. For the same excluded volume (systems I, II, and IV), the superfluid fraction is greater when the number of cylinders is smaller, if the diameter of the hard spheres is comparable to that of the cylinders $(d \sim \sigma)$.

To obtain an estimate of the superfluid transition temperature for each system, we perform a finite size scaling analysis by fitting $N_{s}^{1 / 3} \rho_{s} / \rho$ with [16]:

$$
A+B N_{s}^{(1 / 3 \nu)}\left(T-T_{c}\right)+C N_{s}^{-\Delta /(3 \nu)} \text {. }
$$

This expression assumes the exponents for the superfluid density and the correlation length are related by $(\varphi=$ $-\nu)$. The fit determines the critical temperature $T_{c}$ and the exponent for the correlation length $\nu$. We assumed that $\Delta=1 / 2$, but its precise value has very little effect on the results. The range of reduced temperatures $T_{1} \leq T \leq$ $T_{2}\left(T_{1}\right.$ and $T_{2}$ given in Table II), included in the fit was adjusted to ensure that the superfluid density was linear. The fit for system I is given in Fig. 3. Shown in Table II 


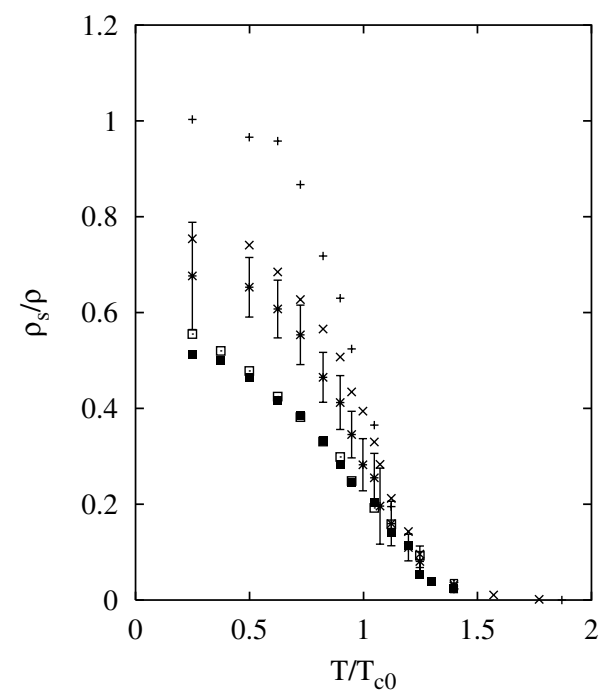

FIG. 2. The superfluid density for the pure system (pluses), system II (crosses), I (stars), IV (open squares), and III (full squares). Here $54 \leq N_{s} \leq 64$. The same ordering of the superfluid density occurred for other values of $N_{s}$. The error bars are shown for one of the systems, and are comparable in all other cases, except in the clean system, in which they are a factor of 2 smaller.

are the transition temperatures obtained with two different fits: one with the $\nu$ 's corresponding to the universality class of the hard spheres (helium), and the other choosing $\nu$ to minimize $\chi^{2}$. Note that $T_{c}$ is independent of the value of $\nu$ chosen. The introduction of the cylinders does not change $T_{c}$ much from a homogeneous ideal gas at the same density. Recall that without disorder $T_{c} / T_{c 0}=$ $1.057 \pm 0.003[1]$ (see Fig. 4).

As the density of cylinders increases (system II vs I) or their diameter increases (system I vs IV), the critical temperature increases. This is most likely because as the available space for the hard spheres decreases, their effective density increases. On the other hand, for the same kind of cylinders (systems II and III), $T_{c}$ does not change when the excluded volume does. Figure 4 shows the comparison with the bulk system. We see that within error bars,

TABLE II. Transition temperature $T_{c}$ and exponent $\nu$ as determined by the fit to Eq. (1). $T_{1} \leq T \leq T_{2}$ is the fit range, $\chi^{2}$ is the reduced fit residual. The number of data points in each fit was 32 .

\begin{tabular}{lccccr}
\hline \hline System & $T_{1} / T_{c 0}$ & $T_{2} / T_{c 0}$ & $T_{c} / T_{c 0}$ & $\nu$ & $\chi^{2}$ \\
\hline I & 0.72 & 1.12 & $1.02 \pm 0.05$ & 0.67 (fixed) & 0.8 \\
I & 0.72 & 1.12 & $1.02 \pm 0.05$ & $0.66 \pm 0.05$ & 0.8 \\
II & 0.72 & 1.20 & $1.15 \pm 0.05$ & 0.67 (fixed) & 1.4 \\
II & 0.72 & 1.20 & $1.15 \pm 0.05$ & $0.71 \pm 0.05$ & 1.4 \\
III & 0.72 & 1.25 & $1.15 \pm 0.20$ & 0.67 (fixed) & 0.7 \\
III & 0.72 & 1.25 & $1.12 \pm 0.75$ & $1.30 \pm 0.5$ & 0.4 \\
IV & 0.62 & 1.25 & $1.17 \pm 0.05$ & 0.67 (fixed) & 14.5 \\
IV & 0.62 & 1.25 & $1.17 \pm 0.17$ & $1.32 \pm 0.08$ & 1.6 \\
\hline \hline
\end{tabular}

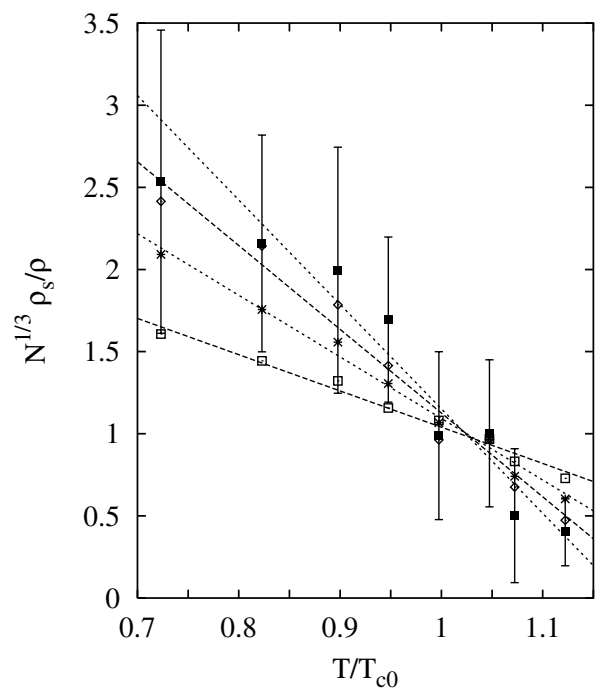

FIG. 3. Scaled superfluid density for system I. The symbols refer to $N_{s}=19,54,99$, and 153 . The dotted lines are the fits and their intersection marks the critical temperature for this system, in this case $T_{c} / T_{c 0}=1.02 \pm 0.05$. The error bars are given for $N_{s}=153$, and are larger at least by a factor of 2 than in the other cases.

disorder does not increase the transition temperature, but on the other hand, it is not reduced either.

With respect to the universality class of the systems under consideration, our conclusions are severely limited by the statistical quality of the parameters obtained from the fit to Eq. (1). For the most disordered system (III), the one with an excluded volume of 0.33 , we cannot draw

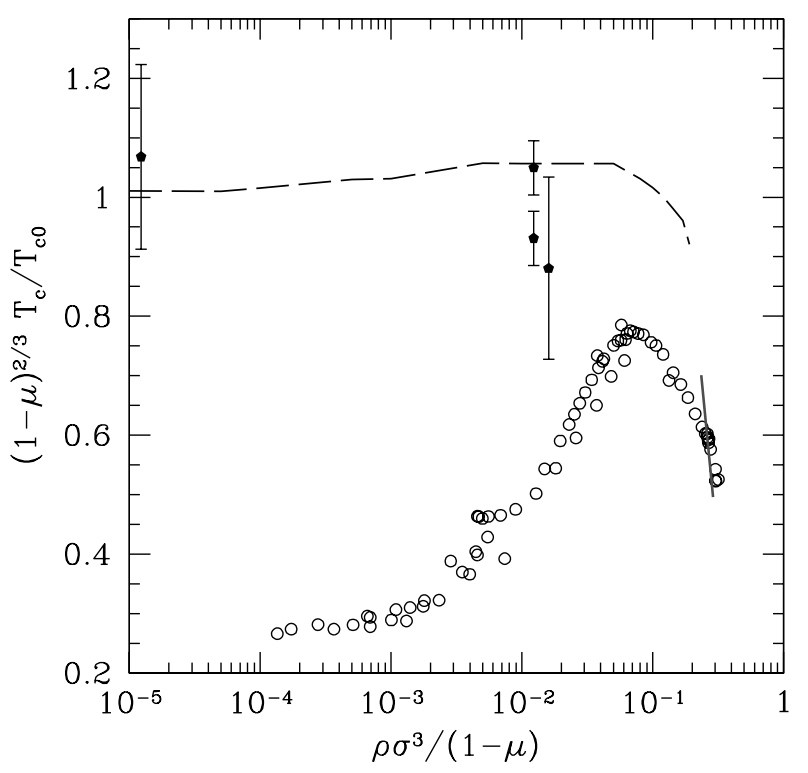

FIG. 4. The superfluid transition temperature versus the density. The dashed line shows the calculations of Ref. [1]. The circles are the data shown in Fig. 3 of Ref. [4]. They have been corrected for the free volume, tortuosity, and effective mass. The solid symbols with error bars are the present calculations. The solid line is for bulk liquid ${ }^{4} \mathrm{He}$. 
any conclusion about the value of $\nu$, so we cannot say if the universality class has changed. Luckily, this fact is irrelevant for the critical temperatures. In the other three systems, the situation is better. The error bars of the critical exponent for the correlation length are small enough to conclude that systems I and II belong to the same universality class as pure helium (for which $\nu=0.67$ ). On the other hand, in system IV $\nu=1.32 \pm 0.08$. This value is clearly different from 0.67 , a result reinforced by the dramatic decrease in $\chi^{2}$ when we go from $\nu=0.67$ to the best fit of $\nu=1.32$ (see Table II). This system might be in a different universality class from pure helium. Such an increase of $\nu$ has been experimentally observed in helium absorbed in aerogel and xerogel $[10,11,13]$.

We now compare these calculations to the measurements of the transition temperature in Vycor. There are several important differences. In Vycor the first helium atoms added to the system are localized on the surface of the silica and do not contribute to a superfluid response (the dead layer). The next helium atoms form a surface layer around the silica strands which is locally 2D but connects up at a longer length scale as a 3D network. The pores are estimated to be in the range of 40 to $80 \AA$. For the sample of Refs. $[4,8]$ we estimate the excluded volume, including that of the dead layer, is $\mu=0.772$. This is a much larger excluded volume factor and a much larger pore size than in our simulations. The tortuosity of Vycor is estimated to have a value of $\chi=0.76 \pm 0.01$ similar to that of our systems.

Figure 4 shows the comparison of the current results with the experiment. To make this comparison, we corrected the densities for the excluded volume in both sets of data. Plotted on the horizontal axis is the density of helium in units of hard-sphere radii, defined as the number of free atoms (not including the dead layer) divided by the free pore volume. The vertical axis is the transition temperature divided by the ideal transition temperature also taking into account the corrected density. The upper curve is the estimate for pure hard spheres of Ref. [1]. The transition temperature in Vycor is reduced from the bulk hard spheres value much more than in our calculation, by up to a factor of 3 , most likely because of the density variation within a pore and between pores. Assuming a pore size of $40 \AA$, for densities such that $\rho \sigma^{3} /(1-\mu) \gtrsim 1.1 \times 10^{-3}$, one has more than 1 free atom/per pore, thus for these densities, Bose condensation can take place inside a single pore. However, not until the pores are nearly full, is our model of strictly repulsive disorder relevant for Vycor because of layering within a pore. This effect, as well as the sig- nificant difference in the ratio of pore size to hard-sphere diameter, likely accounts for the different reductions in the transition temperature at low density. Also plotted in Fig. 4 is the transition temperature of bulk ${ }^{4} \mathrm{He}$ versus density, showing essentially the same reduction as for ${ }^{4} \mathrm{He}$ in Vycor at the same density.

In conclusion, we have performed PIMC simulation to determine the superfluid density for a hard sphere system outside of randomly placed cylinders. We have an overall reduction in the superfluid density, however taking into account the reduced free volume, the transition temperature is reduced only slightly if at all.

This research was supported by NSF DMR-98-02373, NCSA for computer facilities, and the Department of Physics at the University of Illinois Urbana-Champaign. One of us (M.C.G.) thanks the Spanish Ministry of Education and Culture for financial support. Comments of J. Reppy, M. Holzmann, F. Laloë, and G. Baym are appreciated.

[1] P. Grüter, D. M. Ceperley, and F. Laloë, Phys. Rev. Lett. 79, 3549 (1997).

[2] M. Holzmann and W. Krauth, Phys. Rev. Lett. 83, 2687 (1999).

[3] M. Holzmann, P. Gruter, and F. Laloë, Eur. Phys. J. B 10, 739 (1999); G. Baym, J. P. Blaizot, M. Holzmann, F. Laloë, and D. Vautherin, Phys. Rev. Lett. 83, 1703 (1999).

[4] J. D. Reppy, B. C. Crooker, B. Hebral, A. D. Corwin, J. He, and G. M. Zassenhaus, Phys. Rev. Lett. 84, 2060 (2000).

[5] Y.H. Li and S. Teitel, Phys. Rev. B 41, 11388 (1990).

[6] K. Moon and S. M. Girvin, Phys. Rev. Lett. 75, 1328 (1995).

[7] A. B. Harris, J. Phys. C 7, 1671 (1974).

[8] B. C. Crooker, B. Hebral, E. N. Smith, Y. Takano, and J. D. Reppy, Phys. Rev. Lett. 51, 666 (1983).

[9] J. D. Reppy, Physica (Amsterdam) 126B+C, 335 (1984).

[10] M. H. W. Chan, K. I. Blum, S. Q. Murphy, G. K. S. Wong, and J. D. Reppy, Phys. Rev. Lett. 61, 1950 (1988).

[11] G. K. S. Wong, P. A. Crowell, H. A. Cho, and J. D. Reppy, Phys. Rev. Lett. 65, 2410 (1990).

[12] P. A. Crowell, F. W. Van Keuls, and J. D. Reppy, Phys. Rev. B 55, 12620 (1997).

[13] J. Yoon, D. Sergatskov, J. Ma, N. Mulders, and M. H. W. Chan, Phys. Rev. Lett. 80, 1461 (1998).

[14] J. Yoon and M.H.W. Chan, Phys. Rev. Lett. 78, 4801 (1997).

[15] D. M. Ceperley, Rev. Mod. Phys. 67, 279 (1995).

[16] E. L. Pollock and K. J. Runge, Phys. Rev. B 46, 3535 (1992). 